\title{
Dynamical PN Evolution with Magnetic Fields
}

\author{
Guillermo García-Segura \\ Instituto de Astronomía, Universidad Nacional Autónoma de México, Apdo Postal 877, \\ Ensenada, Baja California, Mexico
}

\begin{abstract}
Hydrodynamical simulations played an important role in understanding the dynamics and shaping of planetary nebulae in the past century. However, hydrodynamical simulations were just a first order approach. The new millennium arrived with the generalized understanding that the effects of magnetic fields were necessary to study the dynamics of planetary nebulae. Thus, B-fields introduced a whole new number of physical possibilities for the modeling. In this paper, we review observational work done in the last 5 years and several works on the magnetohydrodynamics of proto-planetary nebulae, since all the effort has been focused on that stage, and discuss different scenarios for the origin of magnetized winds, and the binarybipolarity relation.
\end{abstract}

Keywords. MHD — Stars: AGB and Post-AGB - Planetary Nebulae: general

\section{Introduction}

The origin and evolution of proto-planetary nebulae (PPNs) and planetary nebulae (PNs) represents one of the key questions in our understanding of stellar physics. Modeling the fascinating features displayed by these objects requires not only a better knowledge of stellar structure at the AGB stage (and beyond) but also a proper consideration of the driving mechanisms for mass ejection. The transition from AGB to Post-AGB to PN central stars involves drastically different conditions at every stage. Whereas radiation pressure on dust grains is the most likely mechanism at the AGB phase, as are line-driven winds in the case of PN central stars, for Post-AGB stars the details of the driving force has been relatively unexplored.

To begin this review, it is interesting to mention the increasing number of articles related to magnetic fields in PNs during the last years. Before IAU Symp. 180 (Groningen 1996), the average number of papers per year was 0.41 (base line of 39 years). Over the next 5 years until IAU Symp. 209 (Canberra 2001) the number increased to 6.2 per year Over the last 5 years this number had grown to 11.8 per year by the present Symposium (Hawaii 2006). What is even more interesting is that, in the last five years, $75 \%$ of all the papers were observational. Finally, measurements of magnetic field intensities and their orientations have been made!! This fact will be important in the next five years, since theoretical models have something robust to start with.

All the theoretical work done on PNs up to 2001 was focused on the line-driven wind theory, including weak or moderate magnetic fields frozen in the winds (see review by García-Segura 2003). However, since the paper by Bujarrabal et al. (2001), in which it is stated that PPNs could not be explained by radiation forces on the winds, most of the work done in the last five years has focused on PPNs and the winds from post-AGBs stars. 
In this paper, we first make a short review of the observations done in the last five years, and then proceed with the MHD work.

\section{Magnetic field measurements}

\section{1. $\mathrm{SiO}, \mathrm{H}_{2} \mathrm{O}$ and $\mathrm{OH}$ masers: Zeeman}

15 objects with circularly polarized masers due to the Zeeman effect have been detected so far: S Per (Vlemmings et al. 2001, 2002, 2005); K 3-35 (Miranda et al. 2001, Gómez et al. 2003, 2005); RT Vir, RCrT, W Hya (Szymczak et al. 2001); VY CMa, NML Cyg, U Her (Vlemmings et al. 2002, 2005); W43A (Imai et al. 2002, Vlemmings et al. 2006); OH 17.7-2.0 (Bains et al. 2003, Szymczak \& Gérard 2004); U Ori, VX Sgr (Vlemmings et al. 2005); IRAS 20406+2953 (Bains et al. 2004); IRAS 07331+0021, IRAS 18266-1239 (Szymczak \& Gérard 2004). The measurements for the magnetic fields intensities go from $10^{-2}$ gauss for $\mathrm{OH}$ masers up to several tens of gauss for SiO masers (Vlemmings et al. 2005, 2006). The most exciting object is W43A, which presents a precessing, magnetically collimated jet (Vlemmings et al. 2006), showing also how the field is oriented in the flow. These measurements will constrain future MHD models of winds in late AGB stars, and disk-wind models from interacting binaries and common envelope phases.

\subsection{Nebulae with toroidal fields: dust grain alignments}

The alignment of dust grains under the influence of a magnetic field can be observed in linear polarization in the submillimetre regime. The following nebulae show toroidal field configurations: NGC 7027 and CRL 2688 (Greaves 2002, Sabin et al. this volume); NGC 6537 and NGC 6302 (Sabin et al. this volume).

\subsection{Central stars of PNs: Zeeman}

Magnetic fields of the order of kilogauss have been finally detected in central stars of PNs. The detected objects are NGC 1360, EGB 5, LSS 1362 and Abell 36 (Jordan et al. 2004, 2005). Those central stars are hot and evolved; thus, these measurements will constrain MHD models with line-driven, magnetized winds.

\subsection{Large scale fields: Faraday rotation in PNs}

The discovery of a Faraday screen feature associated with a known astronomical object, the PN S216, has been reported by Uyaniker (2004). This detection is extremely interesting for two reasons, the first one is that it is the first Faraday screen in which the distance is known $(\mathrm{d} \sim 80 \mathrm{pc})$, and the second one is that it proves the existence of large scale magnetic fields associated with a $\mathrm{PN}\left(\mathrm{S} 216\right.$ is $3 \times 3 \mathrm{pc}, 1^{\circ}$ in the sky).

\section{The PPN-wind problem: radiation ruled out}

Winds from AGB stars are thought to be driven by radiation pressure on dust grains (see review by Habing 1996), although an alternative physical mechanism has been proposed by Pascoli (1997) based on magnetic pressure that is transported out from the stellar interior to the stellar surface. On the other hand, it is widely accepted that planetary nebulae are powered by line-driven winds emerging from their central stars, and they are formed from a two-wind dynamic interaction (i.e., Kwok, Purton \& Fitzgerald 1978). Evidence for this scenario includes the large number of P-Cygni line profiles detected in their central objects (Perinotto 1983). 
Post-AGB stars with their associated Proto-planetary nebulae (PPNs) are short-lived transition objects between AGB stars and white dwarfs. Their wind energy source, although unclear, has been usually assumed to be radiation pressure. However, recent observations of PPNs (Alcolea et al. 2001; Bujarrabal et al. 2001 and references therein) have revealed that the linear momenta and kinetic energies associated with these objects are in excess to what can be provided by radiation pressure alone, in some cases by up to three orders of magnitude. These large amounts of momentum and energy, as discussed in detail by Bujarrabal et al. (2001), cannot be accounted for by either radiation pressure on dust grains, line-driven winds or continuum-driven winds.

\section{Magnetic-driven winds: a possible solution}

Four scenarios with different types of wind driving mechanisms have been suggested in the literature:

\section{A. Accretion disk wind solutions}

The theory of magneto-centrifugal launched winds from an accretion disk, where toroidal magnetic fields become finally dominant (e.g. Contopoulos 1995) is applied in this scenario. Several solutions differ in the accretion mode: accretion of the primary wind onto the secondary (e.g. Morris 1987, Mastrodemos \& Morris 1998); Roche lobe overflow (e.g. Livio, Salzman \& Shaviv 1979; Livio \& Soker 1988); accretion on the primary after common envelope evolution (in this case, the disk is formed around the primary star). Recent works on this scenario are Reyes-Ruiz \& López (1998), Blackman et al.(2001b), Frank \& Blackman 2004, and Frank (this volume).

\section{B. Stellar (Post-AGB) solutions}

The results discussed by Pascoli (1997), based on surface magnetic pressure as the main driver of the large mass-loss rates in AGB stars, are an alternative to generate the required mechanical power in the winds of Post-AGB stars (García-Segura et al. 2005), provided that the generation of magnetic fields can be efficient in post-AGB stars, as suggested by Blackman et al. (2001). There is not yet a clear model of how a single star can achieve this. One plausible scheme is that the rotation rate and the field strength at the stellar core increase during the formation of the white dwarf. Thus, the inner magnetic field becomes stronger as the core contracts and becomes exposed at the stellar surface when the envelope is peeled-off during the PN formation. Thus, a strong and dominant toroidal component develops at the interface between the core and the envelope, where some dynamo action is expected and which may be responsible for launching a magnetically driven wind. Actually, Blackman et al. (2001), Matt, Frank \& Blackman (2004) and Miyaji et al. (this volume) have proposed that the post-AGB wind is produced by magneto-centrifugal processes, when dynamo activity increases the internal field (see Blackman 2004) and the AGB star sheds its outer layers, exposing the rotating and magnetized core. Obviously, more detailed stellar interior studies with rotation and $B$ fields are needed to understand the details of this issue. In addition, some authors have suggested that this may also occur in binary systems and, for instance, Soker (1997) proposed that the Post-AGB stellar core can be spun-up by a secondary, increasing the shear between the core and the envelope.

\section{Stellar + disk solutions}

This scenario combines the two solutions above, for the case in which the disk is formed around the primary star (Blackman et al. 2001; Frank, this volume). 


\section{Dynamo during common envelope phase}

The spiral-in process of a secondary star, or a giant planet, may also be able to produce a large shear in the stellar envelope, and raise the magnetic field strength by dynamo activity (Tout \& Regös 2003). These cases link the large mass-loss rates in the post-AGB stages with a common envelope phase. Unfortunately, there are very few detailed studies of post-common envelope systems (e.g. Exter, Pollaco \& Bell 2003).

\section{Magneto-centrifugal core-envelope launching}

A promising avenue, using dynamo amplification at these late evolutionary stages, has been discussed by Matt et al. (2004). They use a simplified model in which the interface between the (rotating and magnetized) stellar core and envelope stores a large amount of magnetic energy due to the twisting of an originally poloidal magnetic field. The magnetic energy is extracted from the stellar rotational energy, causing a rapid spin-down of the proto white dwarf, and is able to drive a strong and short outburst (this is somehow similar to the "magnetic bubble" mechanism proposed by Draine (1983), to generate molecular outflows in star-forming clouds). The outflow can expel the envelope and is termed "magnetic explosion" by Matt et al. (2004; see also the work by Miyaji et al. in this volume).

\section{Proto-Planetary Nebula MHD Models}

García-Segura et al. (2005) have followed magnetic-driven wind expansion and nebula formation for six models. Three of them, models A, B and C have a spherically symmetric initial atmosphere (their Figure 1), while models D, E and F (their Figure 2) have an equatorial density enhancement.

The numerical solutions show that collimation is well established at the very early phases of evolution, creating jet-like outflows at locations close to the star. The inclusion of the density enhancement (their Figure 2) produces, as expected, a narrow equatorial waist without any apparent direct impact at the polar regions. The polar expansion velocities are similar for all models with the same input magnetic field; models A and D have $v_{\text {exp }} \sim 30 \mathrm{~km} \mathrm{~s}^{-1}$, models B and E have $v_{\text {exp }} \sim 150 \mathrm{~km} \mathrm{~s}^{-1}$, while models $\mathrm{C}$ and $\mathrm{F}$ have $v_{\text {exp }} \sim 390 \mathrm{~km} \mathrm{~s}^{-1}$.

As a comparative example, their Figure 3 compares the result of one of the models with 1 gauss (model E) at 1,000 yr, with two well known, extremely collimated PPNs, He 3-401 (Sahai 2002) and M 2-9 (Schwarz et al. 1997). It is apparent in this figure that the solution is able to reproduce convincingly the extreme collimated shapes, along with the sizes and kinematics of these nebulae. Similar results also have been computed by Washimi et al. (this volume).

We now turn our attention to the kinetic energy and linear momentum contained in the outflows from these models. Bujarrabal et al. (2001), as mentioned earlier, have pointed out that radiation pressure is insufficient to provide the observed mechanical power in the outflows of PPNs. Figure 6 of García-Segura et al. (2005) gives the results for three different values of the surface magnetic field covering the initial 1000 years of evolution. The data on PPNs from Bujarrabal et al. (2001) are indicated as crosses in these plots. The values for most of these objects seem to be well bracketed by models B (1 G) and C (5 G). Therefore, magnetic-driven winds are able to provide the necessary energy budget to power the outflows of PPNs. 


\section{Magnetic Cycles}

Magnetic cycles, and their associated field reversals, have been proposed as a plausible origin for the multiple, regularly spaced, and faint concentric shells around some planetary nebulae observed with the Hubble Space Telescope (Soker 2000; García-Segura et al. 2001). In fact, OH maser observations by Szymczak et al. (2001) suggest that changes in the polarized maser emission in some stars could be caused by turbulence in the circumstellar magnetic field and by global magnetic field reversals.

García-Segura et al. (2005) explored the effects of magnetic field reversals in magneticdriven winds, and compared the results with objects displaying collimated outflows with periodic outburst features. An interesting example is He 2-90, a PPN whose symmetric and highly collimated, knotty, bipolar outflow was described by Sahai \& Nyman (2000). The radial velocities of the knots have been measured by Guerrero et al. (2001), and the corresponding proper motions subsequently derived by Sahai et al. (2002). An interesting, and puzzling characteristic in this case is that the collimated outflow, or jet, maintains a nearly constant apparent width throughout all its extent, i.e. it does not fan out at large distances from the star, and the velocity of the regularly spaced knots seems to be the same. The "jet" speed is somewhere between 150-360 $\mathrm{km} \mathrm{s}^{-1}$, its dynamical time is at least $1400 \mathrm{yr}$, and the knots are created at the rate of one pair roughly every 35-45 yr. An extensive numerical study can be found in Lee \& Sahai (2004), who concluded that the inclusion of a magnetic field was necessary.

\section{Bipolar and elliptical nebulae: the role of binaries}

The morphology and galactic distribution of PNs was discussed by García-Segura et al. (2002). We here review the important points.

During the last decade, two important surveys were carried out on both hemispheres. For the southern hemisphere, The ESO Survey, the study was published in a series of papers by Schwarz, Corradi \& Melnick (1992), Stanghellini, Corradi \& Schwarz (1993; 250 PNs), Corradi \& Schwarz (1995), and Corradi (2000; 400 PNs). For the northern hemisphere, The IAC Survey was published by Manchado et al. (1996; $243 \mathrm{PNs}$ ) and Manchado et al. $(2000 ; 255 \mathrm{PNs})$. The first detailed study of the differences between ellipticals and bipolars was done by Corradi \& Schwarz (1995) with the ESO Survey (see also Corradi 2000). They found that the bipolar class has a smaller scale height, $130 \mathrm{pc}$, than that for ellipticals, 320 pc. Also, bipolars have the hottest central stars among $\mathrm{PNe}$, and display smaller deviations from pure circular Galactic rotation than other morphological types. In addition, bipolars also display the largest physical dimensions and have expansion velocities of up to an order of magnitude above the typical values for PNe. These properties, together with the chemical abundance results by Calvet \& Peimbert (1983), indicate that bipolar PNe are produced by more massive progenitors than the remaining morphological classes.

Table 1 .

\begin{tabular}{lc}
\hline \hline $\begin{array}{l}\text { Morphological Class } \\
\text { (IAC Survey) }\end{array}$ & $<z>$ \\
\hline $\mathrm{B}$ & 110 \\
$\mathrm{BPS}$ & 248 \\
$\mathrm{E}$ & 308 \\
$\mathrm{EPS}$ & 310 \\
$\mathrm{R}$ & 753 \\
\hline \hline
\end{tabular}


The average scale height over the plane, $\langle z\rangle$, for different PN morphological classes can be compared with those of different stellar masses and populations. For instance, we know that massive stars are located much closer to the galactic plane than the population of stars with lower initial mass. The outcome from the ESO and IAC surveys is certainly coincident: the bipolar (B) class has $\langle z\rangle=130 \mathrm{pc}$ (ESO) and $\langle z\rangle=179 \mathrm{pc}$ (IAC), for ellipticals $(\mathrm{E})<z\rangle=320 \mathrm{pc}$ (ESO) and $\langle z\rangle=308 \mathrm{pc}$ (IAC), and for round nebulae $(\mathrm{R})<z>=753 \mathrm{pc}(\mathrm{IAC})$.

In the recent analysis of the IAC Survey by Manchado et al. (2000), the bipolar and elliptical objects with point-symmetric features (BPS and EPS) were separated from those which do not present such kind of symmetries, i.e., from the B and E classes respectively. The new results from the IAC Survey are given in Table 1.

Comparing the results of the IAC Survey with those described by Miller \& Scalo (1979) for the average scale height of stars with different masses, an average value equal or smaller than $110 \mathrm{pc}$ corresponds to stars with initial masses above $1.9 \mathrm{M}_{\odot}$. Lower mass stars have average scale heights well above this value. These results are in line with the pioneering suggestion of Calvet \& Peimbert (1983) and the more recent discussion made by García-Segura et al. (1999).

The relation between morphology and galactic distribution was explained by GarcíaSegura et al. (2002) as :

- Bipolars Type I Peimbert $=(B)$ :

Small $<z>(110 \mathrm{pc}) \Longleftrightarrow$ Massive Progenitor $\Longleftrightarrow$ Stellar Rotation $\Longleftrightarrow \Omega$ Limit $\Longleftrightarrow$ Classical Bipolarity

- Bipolars with Point-Symmetry = (BPS):

Moderate $\langle z\rangle(248 \mathrm{pc}) \Longleftrightarrow$ Non-Massive Progenitor in Tidally interacting Binary System $\Longleftrightarrow$ Tidal Spin-Up $\Longleftrightarrow$ Shaping by $\Omega$ Limit + MHD Effects + Precession/Wobbling $\Longleftrightarrow$ Bipolarity with Point-Symmetry (Lobes, FLIERS, Jets)

- Ellipticals $=(\mathrm{E})$ :

Medium $<z>(308$ pc $) \Longleftrightarrow$ Non-Massive Progenitor $\Longleftrightarrow$ Shaping by MHD Effects $\Longleftrightarrow$ FLIERS \& Jets with Axisymmetry

- Ellipticals with Point-Symmetry = (EPS):

Medium $<z>(310 \mathrm{pc}) \Longleftrightarrow$ Non-Massive Progenitor in Wide Binary System $\Longleftrightarrow$ Shaping by MHD Effects + Precession $\Longleftrightarrow$ FLIERS \& Jets with Point-Symmetry

- Round $=(\mathrm{R})$ :

Large $<z>(753 \mathrm{pc}) \Longleftrightarrow$ Low-mass Progenitor $\Longleftrightarrow$ Neither Rotation nor MHD effects

To conclude, the direct signature of a binary is well traced in nebulae with any kind of point-symmetry (BPS and EPS classes), however, the direct link between binarity and bipolarity cannot be concluded (the EPS class is the counter example) from the galactic distribution. It is clear that bipolars are associated with more massive stars. If binaries play a role in the formation of bipolars, at least one or both stars in the binary system must be massive.

\section{Acknowledgements}

G.G.-S. thanks partial support by CONACyT grant 43121. 


\section{References}

Alcolea, J., Bujarrabal, V., Sánchez-Contreras, C., Neri, R., \& Zweigle, J. 2001, A\&A, 373, 932

Bains, I., Gledhill, T. M., Yates, J. A., \& Richards, A. M. S. 2003, MNRAS, 338, 287

Bains, I., Richards, A. M. S., Gledhill, T. M., \& Yates, J. A. 2004, MNRAS, 354, 529B

Blackman, E. G. 2004, in Asymmetric Planetary Nebulae III, ed. M. Meixner, J. Kastner and N. Soker, ASP Conf. Ser., 313, 401 (astro-ph/0309752)

Blackman, E. G., Frank, A., Markiel, J. A., Thomas, J. H., \& Van Horn, H. M. 2001, Nature, 409, 485

Bujarrabal, V., Castro-Carrizo, A., Alcolea, J., \& Sánchez-Contreras, C. 2001, A\&A, 377, 868

Calvet, N. \& Peimbert, M. 1983, RMxAA, 5, 319

Contopoulos, J., 1995, ApJ, 446, 67

Corradi, R. L. M. 2000, in Asymmetrical Planetary Nebulae II: From Origins to Microstructures, eds. Joel H. Kastner, Noam Soker, Saul A. Rappaport, ASP Conf. Ser., 199, 25

Corradi, R. L. M. \& Schwarz, H. E. 1995, A\&A, 293, 871

Draine, B. 1983, ApJ, 270, 519

Exter, K. M., Pollacco, D. L., \& Bell, S. A. 2003, MNRAS, 341, 1349

Frank, A. \& Blackman, E. G. 2004, ApJ, 614, 737

García-Segura, G., Franco, J., López, J. A., Langer, N., \& Rózyczka, M. 2002, RMxAC, 12, 117

García-Segura, G., López, J. A., \& Franco, J. 2005, ApJ, 618, 919

García-Segura, G., López, J. A., \& Franco, J. 2001, ApJ, 560, 928

García-Segura, G. 2003, Proc. IAU Symp. $N^{\circ}$ 209, Planetary Nebulae: Their Evolution and Role in the Universe, eds. S. Kwok, M. Dopita, and R. Sutherland, ASP, p.457

García-Segura, G., Langer, N., Różyczka, M., \& Franco, J. 1999, ApJ, 517, 767

Gómez, Y., Miranda, L. F., Anglada, G., \& Torrelles, J. M. 2003, Proc. IAU Symp. $N^{o} 209$, Planetary Nebulae: Their Evolution and Role in the Universe, eds. S. Kwok, M. Dopita, and R. Sutherland, ASP, p.263

Gómez, Y., Tafoya, D., Anglada, G., Franco-Hernndez, R., Torrelles, J. M., \& Miranda, L. F. 2005, MmSAI, 76, 472

Guerrero, M., Miranda, L.F., Chu, Y-H., Rodríguez, M., \& Williams, R.M., 2001, ApJ, 563, 883

Greaves, J. S. 2002, A\&A, 392, L1

Habing, H. J. 1996, A\&A Rev., 7, 97

Imai, H., Obara, K., Diamond, P. J., Omodaka, T., \& Sasao, T. 2002, Nature, 417, 829

Jordan, S., Werner, K., \& O'Toole, S. J. 2005, A\&A, 432, 273

Jordan, S., Werner, K., \& O'Toole, S. J. 2005, ASPC, 334, 257

Kwok, S., Purton, C. R., \& Fitzgerald, P. M. 1978, ApJ, 219, L125

Lee, C.-F. \& Sahai, R. 2004, ApJ, 606, 483

Livio, M., Salzman, J., \& Shaviv, G. 1979, MNRAS, 188, 1

Livio, M. \& Soker, N. 1988, ApJ, 329, 746

Manchado, A., Guerrero, M., Stanghellini, L., \& Serra-Ricart, M. 1996, The IAC Morphological Catalog of Northern Galactic Planetary Nebulae, ed. Instituto de Astrofísica de Canarias

Manchado, A., Villaver, E., Stanghellini, L., \& Guerrero, M. A. 2000, in Asymmetrical Planetary Nebulae II: From Origins to Microstructures, eds. Joel H. Kastner, Noam Soker, Saul A. Rappaport, ASP Conf. Ser., 199, 17

Mastrodemos, N. \& Morris, M. 1998, ApJ, 497, 303

Matt, S., Frank, A., \& Blackman, E. G. 2004, in Asymmetric Planetary Nebulae III, ed. M. Meixner, J. Kastner and N. Soker, ASP Conf. Ser., 313, 449, (astro-ph/0308548)

Miller, G. E. \& Scalo, J. M. 1979, ApJS, 41, 513

Miranda, L. F., Gómez, Y., Anglada, G., \& Torrelles, J. M. 2001, Nature, 414, 284

Morris, M. 1987, PASP, 99, 1115

Pascoli, G., 1997, ApJ, 489, 946

Perinotto, M. 1983, in Proc. IAU Symp. 103, ed. D. R. Flower, Reidel, Dordrecht, p.323

Reyes-Ruiz, M. \& López, J. A. 1998, ApJ, 524, 952

Sahai, R. 2002, RMxAC, 13, 133

Sahai, R. \& Nyman, L.-A. 2000, ApJ, 538, L145 
Sahai, R., Brillant, S., Livio, M., Grebel, E. K., Brandner, W., Tingay, S., \& Lyman, L.-A. 2002, ApJ, 573, L123

Schwarz, H. E., Aspin, C., Corradi, R. L. M., \& Reipurth, B. 1997, A\&A, 319, 267

Schwarz, H. E., Corradi, R. L. M., \& Melnick, J. 1992, A\&A Suppl, 96, 23

Soker, N. 1997, ApJS, 112, 487

Soker, N. 2000, ApJ, 540, 436

Stanghellini, L., Corradi, R. L. M., \& Schwarz, H. E. 1993, A\&A, 276, 463

Szymczak, M., Blaszkiewicz, L., Etoka, S., \& Le Squeren, A. M. 2001, A\&A, 379, 884

Szymczak, M. \& Gérard, E. 2004, A\&A, 423, 209

Tout, C. A. \& Regös, E. 2003, Magnetic Dynamos in Common-Envelope Evolution, in 3D Stellar Evolution, ASP Conf. Ser., Vol 293, eds. S. Turcotte, S. C. Keller \& R. M. Cavallo, p.100

Vlemmings, W., Diamond, P. J., \& van Langevelde, H. J. 2001, A\&A, 375, L1

Vlemmings, W. H. T., Diamond, P. J., \& van Langevelde, H. J. 2002, A\&A, 394, 589

Vlemmings, W. H. T., van Langevelde, H. J., \& Diamond, P. J. 2005, A\&A, 434, 1029

Vlemmings, W. H. T., Diamond, P. J., \& Imai, H. 2006, Nature, 440, 58

Uyaniker, B., 2004, in The Magnetized Interstellar Medium, eds: B. Uyaniker, W. Reich, and R. Wielebinski, Copernicus GmbH, Katlenburg-Lindau, p.71 